J. Phys. IV France 127 (2005) 181-185

(C) EDP Sciences, Les Ulis

DOI: $10.1051 /$ jp4:2005127028

\title{
Étude de la génération d'harmoniques anormales d'ordre élevé à basse intensité
}

\author{
C. Valentin ${ }^{1}$, S. Kazamias ${ }^{1}$, D. Douillet ${ }^{1}$, Th. Lefrou ${ }^{1}$, F. Augé ${ }^{1}$, S. Sebban ${ }^{1}$, \\ $\mathrm{Ph}$. Balcou ${ }^{1}$ et $\mathrm{V}$. Véniard ${ }^{2}$ \\ ${ }^{1}$ Laboratoire d'Optique Appliquée - ENSTA, École Polytechnique, \\ CNRS UMR 7639 - Chemin de la Hunière, 91761 Palaiseau Cedex, France \\ ${ }^{2}$ Laboratoire de Chimie Physique - Matière et Rayonnement, Université Pierre et Marie Curie, \\ 11 rue Pierre et Marie Curie, 75231 Paris Cedex 05, France
}

\begin{abstract}
Résumé. Nous présentons des résultats expérimentaux de spectre d'harmoniques anormales d'ordre élevé générées à basse intensité laser $\left(210^{13} \mathrm{~W} / \mathrm{cm}^{2}\right)$ dans l'argon. Ce spectre ne peut pas être expliqué par le modèle classique à trois étapes qui prédit les caractéristique du spectre . Les ordres observés dans notre mesure vont bien au delà de la limite de la coupure donnée par l'intensité du laser dans le milieu gazeux. De plus, nous n'observons pas les ordres très bas. Nous présentons une étude systématique de ces spectres, ainsi que des simulations numériques issues de la résolution de l'équation de Schrödinger dépendante du temps.
\end{abstract}

\section{INTRODUCTION}

Les harmoniques d'ordre élevé sont générées lors de l'interaction d'un laser intense et ultracourt avec un gaz. Leur spectre présente une forme caractéristique : l'efficacité des premiers ordres perturbatifs décroît rapidement, puis elle reste constante pour les harmoniques constituant le "plateau", enfin elle chute brutalement après la "coupure". Cette forme est prédite par le modèle à trois étapes [1-3]. On en déduit la loi de coupure $N_{\max } \hbar \omega=3,2 U_{p}+I_{p}$ où $U_{p}$ est le potentiel pondérometeur qui dépend linéairement de l'intensité et $I_{p}$ est le potentiel d'ionisation de l'atome considéré. L'ordre théorique maximum est donné par la loi de coupure appliquée à l'intensité de suppression de barrière $\left(I_{B S I}\right)$ qui est l'intensité pour laquelle le champ électrique compense la barrière coulombienne. Depuis une dizaine d'années, ce modèle est bien vérifié expérimentalement. Plusieurs expériences ont néanmoins montré la possibilité d'atteindre des ordres anormalement élevés [4-7], qui peuvent être expliqués en terme d'ionisation non adiabatique ou par des harmoniques générées par des ions.

Le propos de cet article est de présenter les résultats expérimentaux obtenus à l'aide d'un spectomètre très sensible aux courtes longueurs d'onde, permettant d'observer des spectres étendus d'harmoniques. Dans une première partie, nous décrivons le montage expérimental et nous montrons le spectre obtenu pour l'argon. Nous présentons ensuite une étude systèmatique du signal harmonique en fonction de la position du foyer par rapport au milieu gazeux et en fonction de l'énergie du laser fondamental. Finalement, nous proposons un modèle théorique basé sur la génération d'harmoniques par un processus à $\mathrm{N}$ couleurs : fondamental et harmoniques.

\section{EXPÉRIENCE ET RÉSULTATS EXPÉRIMENTAUX}

\subsection{Montage expérimental}

Le montage expérimental utilisé pour générer des harmoniques d'ordre élevé est décrit dans la référence [8]. Le système laser utilisé est un Ti:Sa de cadence $1 \mathrm{kHz}$ produisant dà $1 \mathrm{kHz}$ des impulsionsde durée $35 \mathrm{fs}$, de longueur d'onde centrale $805 \mathrm{~nm}$. L'énergie du faisceau laser peut atteindre $6 \mathrm{~mJ}$ et peut être modifiée à l'aide d'une lame demi-onde placée avant le compresseur. Le faisceau laser est focalisé sous 
vide dans une cellule remplie de gaz rare de longueur variable. Nous pouvons modifier la position du foyer par rapport à l'entrée de la cellule. La pression du gaz dans la cellule peut être ajustée de 0 à 50 torr. Le faisceau harmonique se propage dans la même direction que le faisceau fondamental, il est ensuite analysé à l'aide d'un spectromètre composé d'un miroir torique en or de réflectivité $40 \%$ et d'un réseau en réflexion en incidence rasante comportant $600 \mathrm{tr} / \mathrm{mm}$. Le miroir focalise les harmoniques dans les directions horizontale et verticale. Le réseau est optimisé pour les courtes longueurs d'onde (jusqu'à $8 \mathrm{~nm}$ ). Afin d'éliminer les radiations infrarouges, nous avons placé deux filtres en aluminium de $250 \mathrm{~nm}$ d'épaisseur chacun sur le trajet des faisceaux IR et harmoniques. Ces filtres ne transmettent les radiations XUV qu'entre 17 et $80 \mathrm{~nm}$ (coupures de l'aluminium), donc il n'est pas possible de détecter les ordres harmoniques supérieurs à 47 et inférieurs à 11 . Leur transmission a été mesurée à $20 \%$ entre 30 et $70 \mathrm{~nm}$ [13]. Le spectre des harmoniques est ensuite enregistré sur une caméra CCD XUV refroidie, avec un temps d'intégration de $1 \mathrm{~s}$. Les spectres sont spectralement calibrés considérant les coupures de l'aluminium à 17 et $80 \mathrm{~nm}$.

\subsection{Spectres expérimentaux}

Le spectre d'harmoniques présente une forme caractéristique : après une diminution régulière du signal, le plateau formé d'harmoniques d'intensité très peu différente est suivi d'une coupure abrupte du signal. Dans le régime de "limite d'absorption", les spectres sont dominés par quelques harmoniques pour lesquelles la longueur d'absorption est grande par rapport à la longueur du milieu [9]. Sur la figure 1, nous montrons le spectre obtenu pour une cellule remplie de 25 torr d'argon. L'intensité estimée était d'environ $210^{13} \mathrm{~W} \mathrm{~cm}^{-2}$ dans le milieu avec une incertitude d'un facteur 2 à cause des variations locales d'intensité. Pour cette intensité, on s'attend à une coupure pour l'harmonique 11.

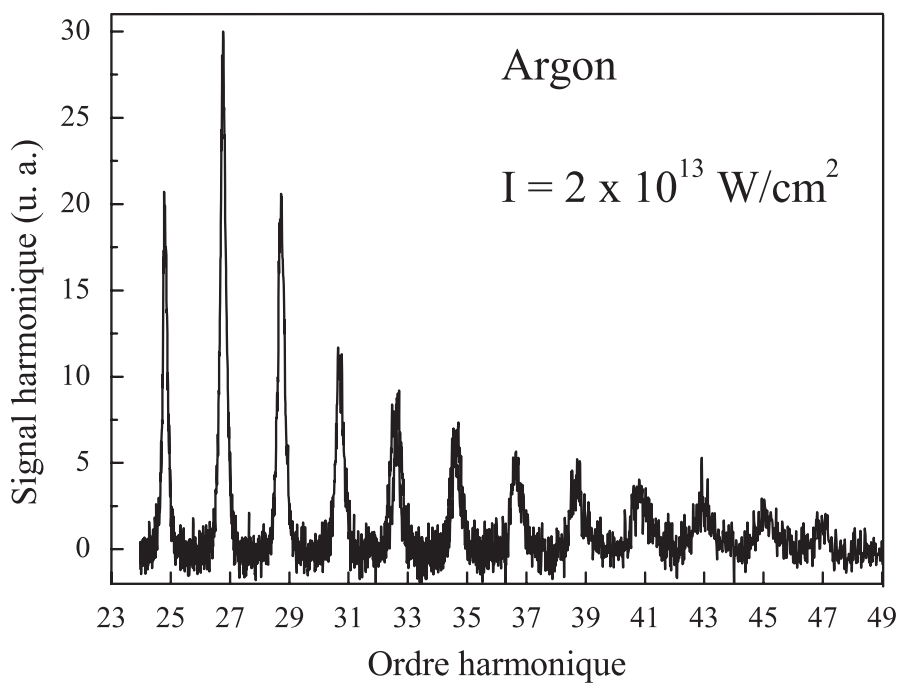

Figure 1. Spectre obtenu avec une cellule de $2 \mathrm{~mm}$ remplie avec 25 torr d'argon. L'énergie du laser était de 3,6 mJ, le diamètre de l'iris de $40 \mathrm{~mm}$ (zone de Rayleigh =2,5 mm), l'entrée de la cellule était placée à environ $25 \mathrm{~mm} \mathrm{du}$ foyer du laser, menant à une intensité dans le milieu de $210^{13} \mathrm{~W} \mathrm{~cm}^{-2}$.

Ce spectre présente un maximum pour l'ordre 27, puis le signal décroît jusqu'à l'ordre 47, correspondant à la limite de détection de notre montage. Un spectre similaire a été enregistré pour le Xénon et il montre une forme analogue avec en plus un deuxième plateau $[12,13]$. Dans les deux cas, nous avons observé des harmoniques bien au delà de la coupure. Nous avons estimé que le nombre maximum de photons par harmonique et par tir est de 20 pour l'harmonique 27 , ce qui est très faible 
comparé au nombre de photons que l'on peut obtenir pour une source harmonique à $35 \mathrm{~nm}$ [9]. La mesure des faibles valeurs de ces signaux a été permise par notre système de détection. Les processus physiques responsables de cette forme de spectre harmonique ne sont alors pas très probables.

Afin de comparer les spectres expérimentaux et théoriques, nous avons tenu compte des facteurs expérimentaux (transmission du gaz, transmission des filtres et efficacité de diffraction du réseau), qui modifient les signaux harmoniques détectés (figure 3a). Nous voyons sur cette figure que le rapport entre le signal d'une harmonique très élevée par rapport à celui d'une harmonique basse est augmenté d'un facteur $10^{4}$ lorsque l'on tient compte des facteurs expérimentaux. Le système de détection que nous avons mis en oeuvre est alors mieux adapté pour mesurer les courtes longueurs d'onde.

\section{3 Étude systématique}

Afin de mieux comprendre ce spectre surprenant, nous avons réalisé une étude systématique. Sur la figure 2a, nous présentons le comportement de deux harmoniques (H33 et H45) générées dans l'argon en fonction de la position du foyer par rapport à l'entrée de la cellule. Le signal harmonique de l'ordre 33 est maximal lorsque le foyer est situé à l'entrée de la cellule. Au foyer, l'intensité est assez importante (2,8 $10^{14} \mathrm{~W} / \mathrm{cm}^{2}$ dans le vide). L'ordre de coupure correspondant est dans ce cas l'harmonique 43 : l'harmonique 33 se situe alors dans le plateau. Ce comportement suit bien la loi de coupure. Par contre, le signal de l'harmonique 45 est faible lorsque l'entrée de la cellule est placée avant ou au foyer, il croît lorsque la cellule est placée après le foyer et il est maximal lorsque l'entrée de la cellule est située à $25 \mathrm{~mm}$ du foyer. L'ordre de coupure est dans cette configuration égal à l'harmonique 15. Le comportement n'est pas symétrique et ne suit pas la loi de coupure.
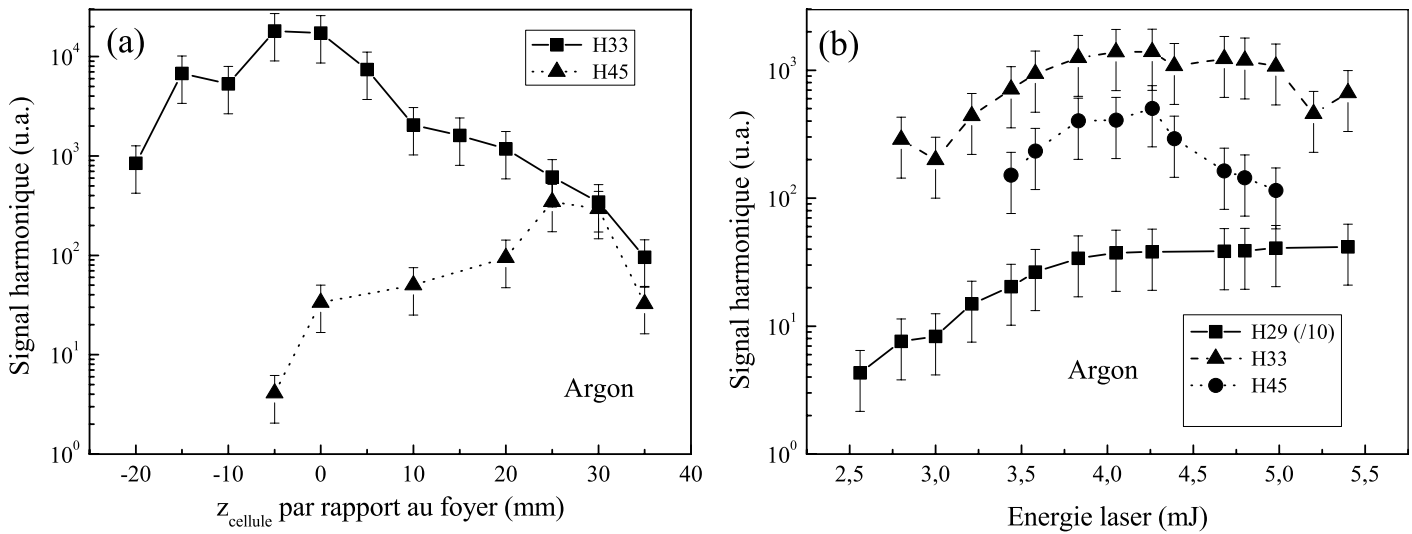

Figure 2. (a) Signal des harmoniques 33 et 45 générées dans une cellule de $2 \mathrm{~mm}$ remplie d'argon en fonction de la position du foyer par rapport à l'entrée de la cellule. L'énergie du laser est 3,45 mJ, le diamètre du diaphragme est $14 \mathrm{~mm}$ (zone de Rayleigh de $11 \mathrm{~mm}$ ) et la pression est de 15 torr. L'intensité estimée varie de $0,310^{14} \mathrm{~W} / \mathrm{cm}^{2}$ $\left(\mathrm{z}_{\text {cell }}=35 \mathrm{~mm}\right) \mathrm{à} 2,810^{14} \mathrm{~W} / \mathrm{cm}^{2}\left(\mathrm{z}_{\text {cell }}=0\right)$. (b) Signal des harmoniques 29 (divisé par 10), 33 et 45 générées dans une cellule de $2 \mathrm{~mm}$ remplie d'argon en fonction de l'énergie du laser fondamental. L'entrée de la cellule est placée 22 $\mathrm{mm}$ après le foyer. L'intensité dans le milieu est estimée à $710^{13} \mathrm{~W} / \mathrm{cm}^{2}$ pour une énergie laser de $4 \mathrm{~mJ}$.

Le comportement des signaux harmoniques en fonction de l'énergie du laser fondamental est encore plus surprenant. En dessous de l'intensité de coupure ( $\mathrm{I}_{c u}$ dépend de l'ordre considéré), le modèle usuel prédit un accroissement exponentiel du signal en fonction de l'intensité [11]. Le signal des harmoniques 29, 33 et 45 générées dans l'argon (figure 2b) augmente lentement avec l'énergie du signal contrairement au comportement attendu pour ces harmoniques. Pour les harmoniques 21 et 45 générées dans le Xénon, nous avons observé un comportement analogue [12]. Ces résultats montrent clairement que le modèle habituel ne peut pas prédire ce spectre à faible intensité et qu'il convient d'élaborer un modèle plus complet. 


\section{RÉSULTATS DE SIMULATION NUMÉRIQUE}

\subsection{Description du code de simulation}

Nous avons résolu numériquement l'équation de Schrödinger dépendante du temps pour un modèle à 1 électron actif. La résolution a été effectuée dans le cas simplifié d'un système à une dimension. Cette approximation permet de décrire correctement les processus physiques mis en jeu dans la génération d'harmoniques, tout en gardant des temps de calcul raisonables. La fonction d'onde de l'électron est discrétisée sur une grille spatio-temporelle et la propagation est effectuée grâce à la méthode dite "split operator" [14]. Le potentiel utilisé pour l'atome est un potentiel Coulombien régularisé : $\mathrm{V}(x)=-\mathrm{Z} /\left(a^{2}+x^{2}\right)^{0.5}$ où $\mathrm{Z}$ est le degré d'ionisation de l'atome ( $\mathrm{Z}=1$ dans notre cas). Le facteur $a$ est ajusté afin de fixer l'énergie de l'état fondamental pour l'atome choisi. Toutes les simulations étant réalisées pour l'atome d'argon, cette énergie est égale à $-\mathrm{I}_{p}(=-15.76 \mathrm{eV})$. Ce code de simulation peut prendre en compte les facteurs expérimentaux calculés. Il est aussi possible de sommer la contribution de plusieurs champs électriques avec des intensités et des pulsations différentes. La figure 3a (traits pointillés) montre un spectre calculé dans l'argon à une intensité de $310^{13} \mathrm{~W} \mathrm{~cm}^{-2}$ sans tenir compte des facteurs expérimentaux. On observe une série d'harmoniques très élevées, dont l'efficacité est très faible par rapport à celle des harmoniques du plateau.
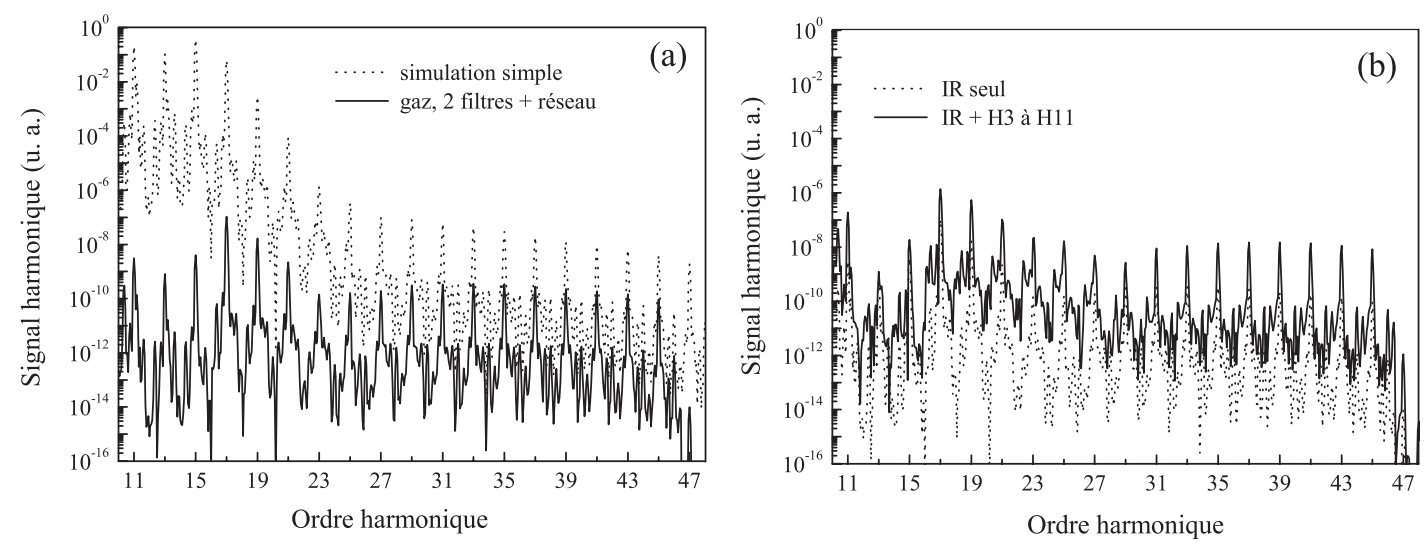

Figure 3. Comparaison entre un spectre calculé pour l'argon à une intensité de $310^{13} \mathrm{~W} / \mathrm{cm}^{2}$ (a) sans correction (traits pointillés) et tenant compte des facteurs expérimentaux (trait plein), (b) avec le champ IR seul (traits pointillés) et avec la somme des champ IR et harmoniques (trait plein), pour les deux spectres on tient compte des facteurs expérimentaux.

\subsection{Harmoniques générées par mélange à $\mathrm{N}$ couleurs}

Afin de comprendre la forme des spectres expérimentaux, nous avons déjà exploré différentes voies [13]. Ces modèles théoriques n'ont pas réussi à expliquer ces spectres, ni la dépendance en fonction de l'énergie du laser fondamental. Nous proposons une nouvelle approche avec des résultats préliminaires encourageants : les harmoniques basses produites au début de la cellule peuvent éventuellement contribuer au champ électrique excitateur. Ce champ s'crit comme une somme des champs électriques IR et harmoniques. On écrit ce champ électrique total comme dans la référence [16], en ne considèrant aucun délai entre le champ IR et les harmoniques en phase. L'enveloppe des champs est trapèzoidale de durée 16 cycles du laser fondamental (correspondant à environ 50 fs). L'intensité du laser fondamental est $310^{13} \mathrm{~W} / \mathrm{cm}^{2}$ et le rapport des intensités pour les différentes harmoniques croissantes est $9,310^{3}$, $310^{3}, 510^{3}, 510^{3}$ pour les harmoniques 3 à 11 respectivement. Sur la figure $3 \mathrm{~b}$, nous voyons que le 
signal des harmoniques d'ordre très élevé est encore augmenté de deux ordres de grandeur lorsque l'on considère les champs harmoniques. Les harmoniques au delà de la coupure (H13 pour cette intensité) peuvent alors être générées par les harmoniques plus basses constituées des ordres situés en dessous de la coupure $(\mathrm{H} 13)$.

\section{CONCLUSION ET PERSPECTIVES}

Nous avons observé et étudié les harmoniques anormales d'ordre élevé générées dans l'argon lorsque le foyer du laser est très éloigné de la cellule de gaz. Le spectre de l'argon pourrait être expliqué à l'aide d'un modèle d'harmoniques générées par les harmoniques plus basses. Les premiers résultats de simulation font apparaître une augmentation très forte de l'efficacité grâce à des processus de couplage d'harmoniques. Une étude plus détaillée en fonction de l'intensité du laser fondamental permettrait de valider ce modèle. De plus, il faudra prendre en compte les effets d'accord de phase qui jouent aussi un rôle dans le processus de génération.

\section{Bibliographie}

[1] K. C. Kulander, K. J. Shafer and J. L. Krause, dans Super-Intense Laser-Atoms Physics (P. Piraux, A. L'Huillier and K. Rzążdewski, New York: PlenumPress, 1993) p 95.

[2] P. B. Corkum, Phys. Rev. Lett. 71, 1994 (1993).

[3] M. Lewenstein, Ph. Balcou, M. Yu Ivanov, A. L'Huillier and P. B. Corkum, Phys. Rev. A 49, 2117 (1994).

[4] C.-G. Wahlström et al., Phys. Rev. A 484709 (1993).

[5] E. Gibson et al., Phys. Rev. Lett. 92, 033001 (2004).

[6] Ivan P. Christovet al., Phys. Rev. Lett. 77, 1743 (1996).

[7] N. A. Papadogiannis et al., Appl. Phys. B 73, 687 (2001).

[8] S. Kazamias et al., Eur. Phys. J. D 21, 353 (2002).

[9] S. Kazamias et al., Eur. Phys. J. D 26, 47 (2003) ; S. Kazamias et al., Phys. Rev. Lett. 90, 193901 (2003).

[10] http://www-cxro.lbl.gov.

[11] M. Lewenstein, P. Salières and A. L'Huillier, Phys. Rev. A 52, 4747 (1995).

[12] C. Valentin et al., Appl. Phys. B. 78, 845 (2004).

[13] C. Valentin et al., J. Phys. B: At. Mol. Opt. Phys. 37, 2661 (2004).

[14] M. H. Hermann and J. A. Fleck, Phys. Rev. A 38, 6000 (1988).

[15] J. H. Eberly, R. Grobe, C. K. Law and Q. Su, dans Atoms in Intense Laser Fields (M. Gravila Academic Press, SanDiego, 1992) p 301.

[16] K. J. Schafer, M. B. Gaarde, A. Heinrich, J. Biegert and U. Keller, Phys. Rev. Lett. 92, 023003 (2004). 